\title{
Dermatología estética facial. Una visión holística
}

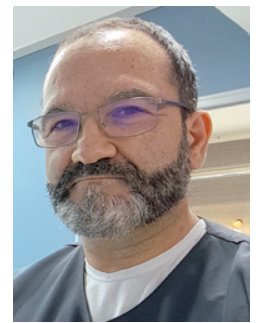

José Ferrandis-Luis Canarias Dermatológica. Las Palmas de Gran Canaria.

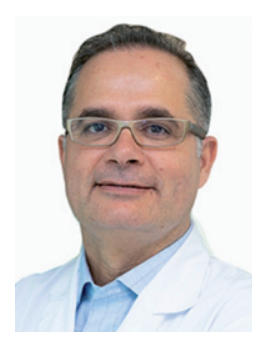

Agustín Viera-Ramírez Canarias Dermatológica. Las Palmas de Gran Canaria.

\section{RESUMEN}

La dermatología estética complementa y potencia la eficacia de la dermatología clínica, al añadir al concepto de salud cutánea el parámetro «bienestar», incluida la idea de «verse bien». Por ello, consideramos al paciente dermoestético como un todo, en una visión integral u holística de su diagnóstico y tratamiento. Esto requiere con frecuencia la participación de otros especialistas — como, por ejemplo, en nutrición, psicología o ecografía - en busca de la salud cutánea en todos sus aspectos.

En el diagnóstico y tratamiento en dermatología estética actual, cobra cada vez más importancia la ecografía cutánea de alta frecuencia, técnica de imagen en consulta que permite objetivar la evaluación del envejecimiento y los tratamientos realizados, reducir las complicaciones, mejorar los resultados y reforzar la confianza del paciente en el proceso.

Igualmente, esa visión holística del paciente lleva cada vez con más frecuencia a protocolos diagnósticos basados en los signos emocionales externos transmitidos por el paciente y producidos por cambios anatómicos identificables, que transmiten emociones no congruentes con las emociones del propio paciente (tristeza, enfado, cansancio, envejecimiento), en lo cual ahondamos ya desde la primera anamnesis, tanto si el paciente acude por una enfermedad dermatológica con repercusión estética, como si busca embellecimiento o rejuvenecimiento. La investigación y la clínica del tratamiento de los atributos emocionales estéticos muestran cómo es capaz de inducir mejoría estética, integrada en la salud del individuo y percibida como «natural», además de mejorar secundariamente su salud emocional.

Dentro de nuestra visión holística de la dermatología estética, analizamos el papel del exposoma, conjunto de factores extrínsecos a los que está expuesto el individuo desde que nace hasta que muere y, en concreto, los que tienen evidencia de acelerar el envejecimiento cutáneo, como determinadas sustancias químicas, la radiación, el calor/frío, el estrés emocional, los hábitos tóxicos y, en especial, la nutrición. Y respecto a esta última, las revisiones recientes muestran indicios de su relación con el envejecimiento extrínseco mediado por el estrés oxidativo, similar al debido al fotoenvejecimiento, pero originado por determinadas pautas nutricionales. Por ello, incluimos el tratamiento nutricional en el tratamiento dermatológico estético, añadiendo, cuando es necesario, el apoyo farmacológico adecuado, como el existente para el control del sobrepeso y la obesidad (tales como el orlistat o, especialmente, la liraglutida) para la mejora de la salud del individuo, lo cual redunda en el aspecto estético de su piel.

Palabras clave: dermatología estética holística, bienestar, ecografía cutánea estética, exposoma, atributos emocionales, nutrición, estrés oxidativo, liraglutida. 


\begin{abstract}
Aesthetic dermatology complements clinical dermatology, enhancing its effectiveness by adding the «well-being» parameter to skin health concept, also including the idea of «looking good». For this reason, we consider the dermaesthetic patient in the diagnosis and treatment as a whole, in an integral or holistic vision. This often requires the participation of specialists in nutrition, psychology, ultrasonography and many other areas, in search of the concept of health in all its aspects, not only the biological one.

In the diagnosis and treatment in current aesthetic dermatology, high frequency skin ultrasound is becoming increasingly important, an in-office imaging technique that allows objectifying the evaluation of aging and the treatments performed, reducing the incidence of complications, and improving results and the patient's confidence in the process.
\end{abstract}

Likewise, we analyze how this holistic view of the patient leads us more and more frequently to diagnostic protocols based on the external signs of emotional attributes, transmitted by the patient, but produced by identifiable anatomical changes and that convey emotions that are not congruent with those felt by the patient himself (sadness, anger, tiredness, flaccidity). We take care of those attributes from the first anamnesis, whether the patient comes for dermatological pathology with aesthetic repercussions, or he is looking for beautification or for rejuvenation. We describe how the treatment of those emotional attributes can induce aesthetic improvement, integrated into the health of the individual, and perceived as «natural», in addition to secondarily improving their emotional health.

Within our holistic vision of aesthetic dermatology, we analyze the role of the exposome, defined as the set of extrinsic factors to which the individual is exposed from birth to death, specifically those that show evidence in accelerating skin aging, such as some chemical products, radiation, heat/cold, stress, toxic habits, and especially nutrition. And regarding the latter, we analyze recent reviews that show signs of extrinsic aging mediated by oxidative stress, in a form and pathways similar to that due to photoaging, but triggered by quantitative and qualitative harmful nutritional guidelines. We describe how we include nutritional treatment as part of aesthetic dermatological treatment, including, when necessary, pharmacological support such as the existing one for the control of overweight and obesity, such as orlistat, or especially liraglutide, for the improvement of the patients' health, which redounds in the aesthetic aspect of their skin.

Key words: holistic aesthetic dermatology, well-being, aesthetic skin ultrasound, exposome, emotional attributes, nutrition, oxidative stress, liraglutide.

Varias son las características que, en nuestra opinión, definen y ayudan a entender la realidad de la dermatología estética actual en nuestro entorno. La primera es que el dermatólogo estético, «como dermatólogo», diagnostica y trata enfermedades cutáneas y, «como estético», va más allá de alcanzar la ausencia de enfermedad: promueve el óptimo estado de salud cutánea, con la visión de que una piel sana es una piel estética. Esta visión, donde la dermatología estética complementa a la dermato- 
logía clínica ${ }^{1}$, y la hace llegar «más allá», se impone en una sociedad cada vez más informada, donde el concepto de bienestar se entiende como estar, saberse sano y «verse bien» ${ }^{2}$.

La segunda característica es que el dermatólogo estético busca alcanzar el máximo bienestar cutáneo posible mediante la inclusión de procedimientos terapéuticos y tecnológicos. Fuentes de luz, dispositivos emisores de energía, peelings químicos o mecánicos, cirugía, infiltraciones de principios activos... forman parte importante de la visión que el dermatólogo estético tiene del tratamiento de procesos dermatológicos y complementan la terapéutica clásica, potenciando su eficacia y mejorando, con ello, sus resultados ${ }^{3,4}$.

La tercera característica de la dermatología estética es su visión humanista y centrada en el paciente, alejada de protocolos diagnóstico-terapéuticos estáticos, en donde no se tiene en cuenta el clásico aforismo del fisiólogo francés de siglo XIX, Claude Bernard, de que «no existen enfermedades, sino enfermos $»^{5}$ y que se favorece por la libertad de que en nuestro entorno se desarrolle la dermatología estética en un contexto de atención privada, no sujeta a cobertura de servicios públicos y a las restricciones que lamentablemente ello genera. Desde la visión de la dermatología estética, abordamos a nuestros pacientes en nuestras consultas mediante perfiles. Un perfil de paciente se define como aquel grupo de pacientes que comparten una serie de características comunes (no solo clínicas, sino también psicológicas, sociales, culturales y ambientales) y que, una vez diagnosticados, nos permite abordarlos terapéuticamente de una forma similar ${ }^{6}$. Esta visión integral de la patología dermatológica (visión holística) hace que, en nuestro equipo de trabajo, los dermatólogos estéticos contemos con distintos profesionales que aportan un valor añadido a nuestros pacientes: médicos nutricionistas, psicólogos, ecografistas, médicos generales, vasculares, plásticos, fisioterapeutas, enfermeras...

En definitiva, la dermatología estética es un área de conocimiento que ha surgido con fuerza dentro de la especialidad médico-quirúrgica de la derma- tología y que se desarrolla en clínicas dermatológicas, con unidades muy tecnológicas, que aportan soluciones individualizadas a problemas dermatológicos, que, sin el trabajo en equipo, tecnología y especialización, no tendrían adecuada solución. La dermatología estética es dermatología personalizada, centrada en el paciente, con una visión integral humanista, a la que se suma la especialización por unidades y el desarrollo tecnológico. No solo trata de ampliar la cartera de servicios o de las innovaciones tecnológicas aplicadas a la dermatología, sino, además, considerar al paciente como el centro de toda la actividad.

Promovemos, por ello, que esta visión de la dermatología estética holística es un «proceso por el que se capacita» a la persona. La salud cutánea no es solo el objetivo último que alcanzar; es un recurso para la vida cotidiana, son herramientas, hábitos, conocimientos... que debemos utilizar en nuestra vida diaria ${ }^{7}$. La dermatología estética busca, con el objetivo de alcanzar la salud cutánea, el empoderamiento del individuo, proceso mediante el cual las personas adquieren un mayor control sobre las decisiones y acciones que afectan a su salud. La dermatología estética es, además, una actitud que adopta el dermatólogo ante su enfermo cuando lo considera y trata como una persona en quien concurren lo biológico o corporal, lo psíquico, lo social y lo cultural, elementos que definen al ser humano y cuyo equilibrio se considera el paradigma de la salud ${ }^{8}$. La dermatología estética busca, en definitiva, que las personas se involucren para que se produzcan cambios en ellas y en su entorno. El cómo motivarlo es uno de nuestros principales objetivos, es parte de nuestra misión.

Dentro de la dermatología estética, el área facial constituye gran parte de nuestra actividad ${ }^{9}$ y es, a partir de una visión holística, integradora, desde donde resaltaremos en este artículo cuatro recientes aportaciones a nuestra práctica clínica. La primera en el área diagnóstica, la segunda en el área de prevención y promoción de la salud facial, y la tercera y cuarta en el área terapéutica. 


\section{DIAGNÓSTICO ECOGRÁFICO FACIAL}

La dermatología estética facial se basa en establecer distintos diagnósticos antes de iniciar cualquier tratamiento. El primero es el diagnóstico cutáneo: descartar enfermedad y evaluar el comportamiento de las distintas células y estructuras que conforman la epidermis-dermis y que influyen en el continuo salud-enfermedad ${ }^{1}$ de la piel (queratinocito, melanocito, fibroblasto, glándula sebácea y vascularización cutánea). Tras el diagnóstico cutáneo, hay que realizar un diagnóstico del rostro estético: simetría, proporciones, características de los tercios faciales y anatomía externa facial, y referencias anatómicas. El tercer diagnóstico es el diagnóstico estático facial, de compartimentos grasos superficiales (nasolabial, intermedio, medial de la mejilla y temporoparietal) y profundos (tejido adiposo suborbicular de los ojos o SOOF [suborbicularis oculi $f a t$ y medial profundo), así como de las características de los ligamentos de retención verdaderos (osteocutáneos) y falsos o retináculo-cutis (fasciocutáneos). La cuarta valoración es el diagnóstico dinámico facial o muscular, evaluando el equilibrio entre contracción y relajación de los distintos grupos musculares, así como su fortaleza. Finalmente, hay que considerar las áreas faciales de alto impacto estético: periocular, labial y nasal.

La ecografía facial estética se ha convertido en una técnica de imprescindible utilidad en la consulta de dermatología convenciona ${ }^{10}$ y dermatología estética ${ }^{11,12}$. La ecografía cutánea es una aplicación concreta de la ecografía de partes blandas, orientada al estudio específico de la piel y posible gracias al desarrollo en los últimos años de sondas ecográficas de alta frecuencia (que alcancen, al menos, $15 \mathrm{MHz}$ ), entre otros requerimientos ${ }^{13}$. Se complementa, entre otros medios, con la información que aporta el estudio Doppler en el mismo examen.

Esta exploración, junto con otras técnicas de imagen ${ }^{14}$, sirve de apoyo al diagnóstico del envejecimiento en dermoestética de cuatro formas principales.

\section{Estimación objetiva del envejecimiento cutáneo}

Complementa y mejora la información clínica de las fotos estéticas clásicas de antes/después, siempre controvertidas por su variabilidad y por su dificultad de obtener en condiciones $100 \%$ repetibles. La ecografía cutánea permite tomar imágenes en condiciones objetivamente idénticas, y obtener indicadores cuantitativos del envejecimiento cutáneo en el área que estamos estudiando o tratando ${ }^{15}$. Esto es posible, básicamente, a partir de dos mediciones ecográficas sencillas de obtener ${ }^{16}$ :

1. Banda hipoecoica subepidérmica (SLEB; del inglés, subepidermal low-echogenic band): es la banda hipoecogénica (más oscura) que aparece en el límite dermoepidérmico, fruto del depósito de productos de glicosilación y vinculados, a su vez, con los procesos de elastosis cutánea, tan relacionados con los procedimientos de fotoenvejecimiento a este nivel. Su medición seriada en el tiempo indica el grado de fotoenvejecimiento presente en el área estudiada y su evolución con nuestros tratamientos ${ }^{17}$.

2. Grosor total (GT): distancia medida ecográficamente desde la superficie al límite dermohipodérmico en el área estudiada.

Para minimizar la relevancia de la diferente estructura cutánea en distintas áreas de la piel, con estos dos parámetros, obtenemos la relación SLEB/GT como índice cuantitativo y repetible para el diagnóstico y seguimiento del envejecimiento cutáneo ${ }^{16}$, con una correlación elevada con la histología del envejecimiento de la piel (fig. 1). De esta manera, podemos acceder a esta información sobre la estructura de la piel envejecida, evitando estudios por biopsia que para tratamientos de dermatología estética no se justificarían.

A modo de ejemplo, en la figura 2, podemos ver ecografías de tres pacientes, con edades separadas 20 años entre sí, donde se aprecia que, conforme la piel envejece, aumenta el grosor de la SLEB y se reduce el GT, aumentando el cociente SLEB/GT como medida cuantitativa del fotoenvejecimiento. 


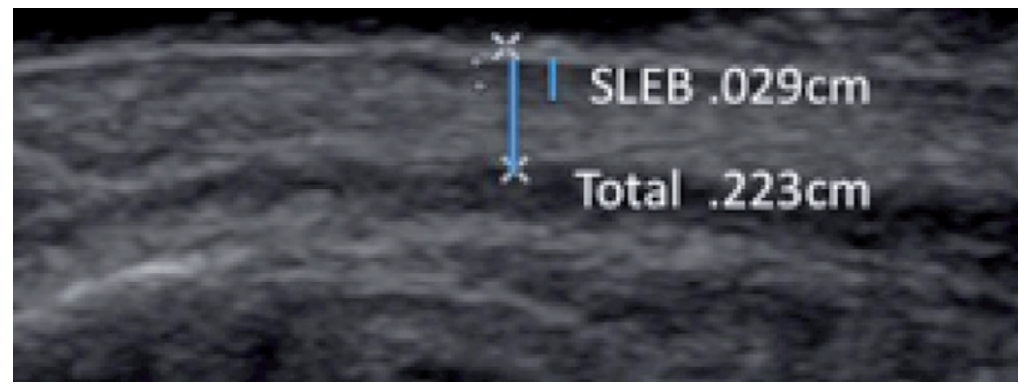

Figura 1. Medición ecográfica de la banda hipoecoica subepidérmica (SLEB; del inglés, subepidermal low-echogenic band) y del grosor total (GT).

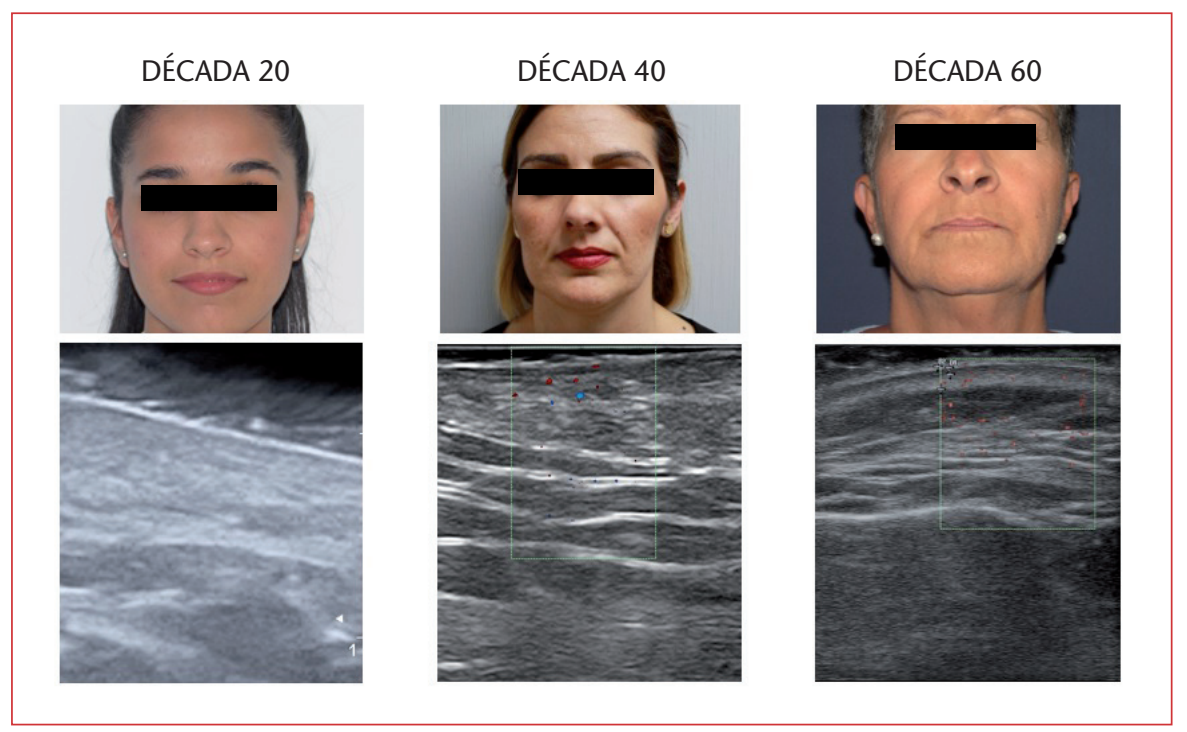

Figura 2. Aumento de la banda hipoecoica subepidérmica (SLEB; del inglés, subepidermal low-echogenic band) y reducción del grosor total en la segunda, la cuarta y la sexta décadas de la vida.

\section{Mapeo de áreas de riesgo vascular}

La región facial es rica en estructuras neurovasculares, algunas con relación topográfica directa con zonas habituales en tratamientos dermoestéticos infiltrativos. Un ejemplo claro es la glabela o zona del entrecejo, donde con frecuencia está indicado infiltrar toxina botulínica para el tratamiento de arrugas dinámicas, o también determinados tipos de ácido hialurónico indicados para técnicas de blanching, o infiltración muy superficial para el componente estático de las arrugas dinámicas. La ecografía permite ${ }^{18}$ conocer y medir si los vasos supratrocleares discurren superficiales en esta zona, y si guardan relación directa con la zona a infiltrar. De esta forma, podremos reducir el riesgo de daños al descartar la técnica, o realizarla con la máxima seguridad para el paciente y para el especialista (fig. 3).

\section{Identificación del paciente idóneo para un tratamiento}

En algunos casos, la estructura anatómica determinante en la aparición de un defecto estético puede no ser la que en principio aparenta. Ello aconseja la identificación por imagen de todas las estructuras musculares en las que esto sea posible ${ }^{19}$. Un ejemplo claro es la protrusión de la zona submentoniana $^{20}$, para la que puede estar indicado el 

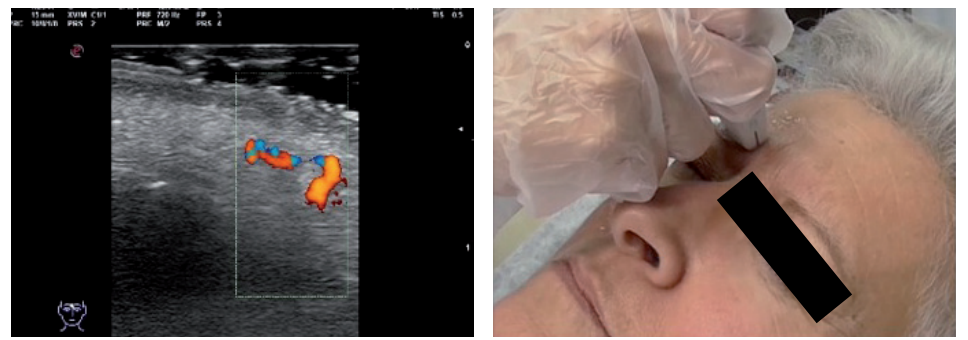

Figura 3. Identificación mediante ecografía de la vascularización en la glabela.

uso de agentes lipolíticos como el ácido desoxicólico $\left(\right.$ Belkyra $\left.^{\circledR}\right)$. Este tratamiento solo resulta eficaz y seguro -y, por lo tanto, solo estará indicado- si la deformidad se debe a un aumento de grasa subdérmica, pero no si se debe a cambios en otras estructuras o compartimentos anatómicos, como por laxitud o hipertrofia de los músculos digástricos, mala posición del hueso hioides, o si se produce a expensas de la grasa subplatismal (profunda), como en el caso de la imagen superior (fig. 4), en la que, mediante ecografía, encontramos que el aumento de volumen se debe a una pseudoherniación de dicha grasa subplatismal hacia planos superficiales. En estos últimos casos, el lipolítico no sería eficaz, y podría dañar la piel o la fascia al infiltrarlo.

\section{Detección de materiales de relleno previos}

De especial utilidad para materiales de relleno permanentes, que a lo largo de la historia de los tratamientos estéticos infiltrativos han tenido uso aprobado o, en algunos casos, aún siguen utilizándose. Esto es posible mediante la identificación de determinados patrones ecográficos ${ }^{21,22}$ en la exploración cutánea ecográfica y, muy especialmente, el patrón vacuolar, patrón en nevada y formas mixtas. La información que ofrece la ecografía en este aspecto es de alta sensibilidad y correlación histológica, y permite disponer de esta información, reduciendo la necesidad de estudios histológicos, salvo en casos dudosos (fig. 5).
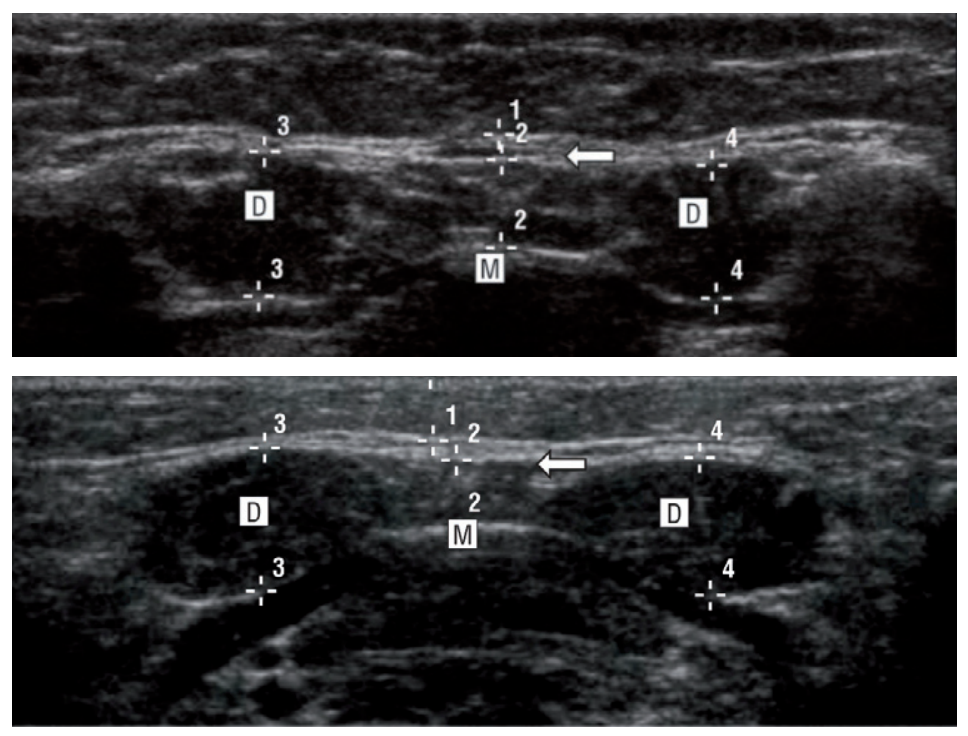

Figura 4. Imagen superior: pseudoherniación de la grasa subplatismal (contraindicación de lipolítico). 


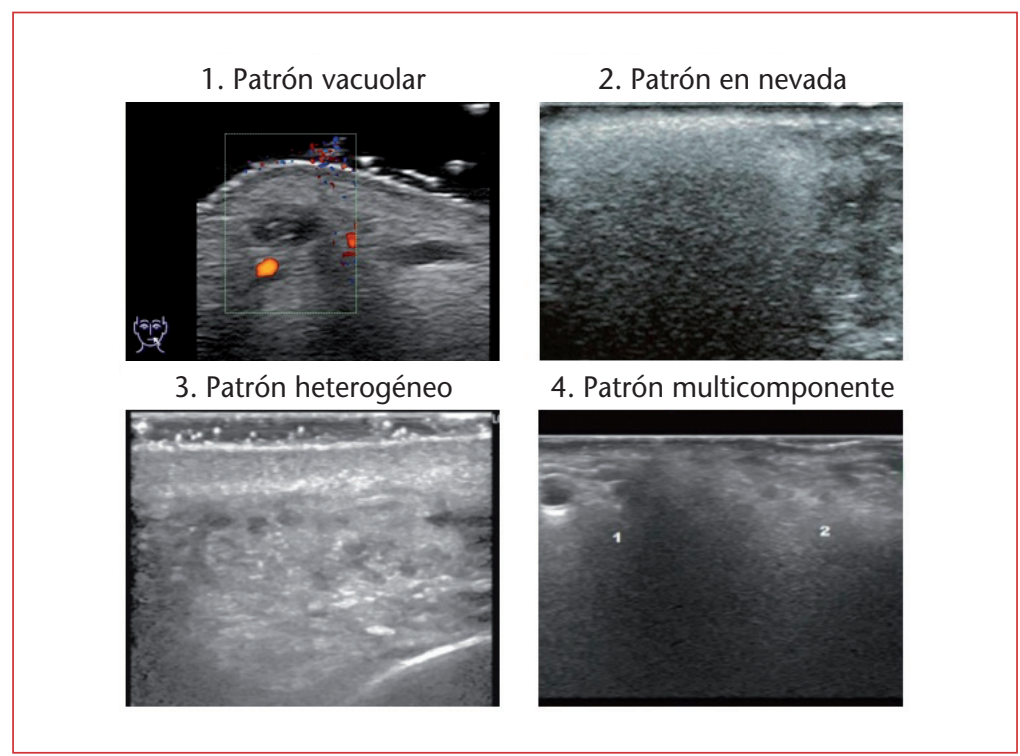

Figura 5. Patrones ecográficos de rellenos permanentes.
Combinando la información de la exploración ecográfica del paciente con la obtenida por la anamnesis de los antecedentes estéticos (tiempo de evolución del relleno y otros), podemos detectar con fiabilidad las áreas faciales donde no es seguro realizar una nueva infiltración, por la probable presencia de rellenos permanentes, e identificar el producto o el tipo de producto presente, en caso de desconocerse. Asimismo, en algunos casos, estos cambios ecográficos impiden conocer la anatomía de la zona, lo cual dificulta o puede contraindicar toda infiltración, por insegura, en esas áreas afectadas ante el riesgo de generar complicaciones en lugar de beneficios, desde la aparición de respuesta inmunitaria local contra ellos, como las reacciones a cuerpo extraño, hasta complicaciones vasculares por no disponer de información concreta sobre la vascularización de zonas comprometidas previamente tratadas. Y no hay que olvidar que la primera obligación ética y legal del médico es no hacer daño al paciente.

\section{EXPOSOMA}

El genoma es el conjunto de genes en los que está contenida toda la información necesaria para de- sarrollar los caracteres del ser humano. El estudio de los genes explica, aproximadamente, el $25 \%$ de las enfermedades crónicas, pero el otro $75 \%$ se explica mediante el concepto de exposoma, término acuñado en 2005 por el científico Christopher P. Wild y definido como el conjunto de factores de exposición a los que está sometida una persona desde el momento de su concepción hasta que muere. Una persona está expuesta a sustancias químicas, radiación, calor/frío, estrés, alimentación, actividades, comportamientos de salud, etc. La suma de estos factores externos actúa sobre el genoma a lo largo de la vida. La importancia de este concepto radica en que se ha postulado que el exposoma puede ser el responsable de múltiples enfermedades crónicas ${ }^{23,24}$.

Recientemente, se ha identificado y se está estudiando el exposoma de la piel $^{25,26}$ en lo que se refiere al envejecimiento de esta ${ }^{27}$, y se han definido siete factores de exposoma externos y modificables que afectan al envejecimiento de la piel facial: radiación solar, polución, tabaco, temperatura, nutrición, estrés, falta de sueño y uso de cosméticos.

El fotoenvejecimiento de la piel humana resulta de la exposición solar diaria no extrema, en baja 
dosis, que no causa cambios visibles en la piel, sino biológicos, celulares. El fotoenvejecimiento afecta a los tres compartimentos cutáneos: epidermis, dermis e hipodermis. Las alteraciones en el compartimento dérmico, a nivel de fibroblastos, es el evento primario. La radiación ultravioleta A especialmente, y los ultravioleta $\mathrm{B}$, son los mayores contribuyentes a este. La susceptibilidad del fotoenvejecimiento está muy influenciada por sistemas de protección endógena presentes en la piel humana tales como la pigmentación, la reparación del ácido desoxirribonucleico (ADN), antioxidantes y otros, que difieren entre individuos por variabilidad étnica, edad o diferencias genéticas. Hay evidencia de que el uso regular de fotoprotectores tópicos puede retrasar el fotoenvejecimiento de la piel humana. Hasta estudios recientes, se pensaba que el fotoenvejecimiento solo estaba producido por la radiación ultravioleta. Actualmente, se sabe que la radiación visible (415 nm y $630 \mathrm{~nm}$ ) modula la pigmentación, y la radiación infrarroja altera la elasticidad de la piel y su firmeza cutánea y facilita la formación de arrugas. La luz visible, la radiación infrarroja y la radiación ultravioleta generan, como comentábamos, fotoenvejecimiento, contribuyendo al daño cutáneo mediante el aumento del estrés oxidativo celular, lo que obliga a adoptar nuevas estrategias de fotoprotección ${ }^{28}$ y a la búsqueda de nuevos antioxidantes externos para luchar contra esta agresión. La mayoría de los fotoprotectores protegen actualmente solo contra las radiaciones ultravioleta, no contra la luz visible e infrarroja.

Más del $97 \%$ de la población urbana en la Unión Europea está expuesta a niveles de partículas físicas y gases que exceden el límite recomendado por la Organización Mundial de la Salud. La contaminación produce un impacto grave en la salud, causando un amplio abanico de daños: aumenta la hospitalización por enfermedades cardiovasculares e insuficiencia cardíaca, disminuye significativamente la función pulmonar y reduce la esperanza de vida. En lo que a la piel se refiere, la contaminación ambiental produce lentigos, arrugas, aumento de la secreción sebácea, aumento de la sequedad cutánea, aumento de la respuesta irritativa de la piel y exacerbación o empeoramiento de trastornos dermatológicos como el acné, la rosácea, el eccema seborreico y otros eccemas.

La relación entre el tabaco y el envejecimiento facial está sustentada por estudios con evidencia científica. El tabaco genera en la piel: 1) piel del fumador: arrugas faciales prominentes, especialmente, alrededor de boca, el labio superior y los ojos; 2) melanosis del fumador: hiperpigmentación, especialmente, en la mucosa oral; 3) irregularidad pigmentaria facial (con mejoría tras su cese); y 4) aumento de arrugas y laxitud.

Un estudio muy interesante, el estudio EPIC (European Prospective Investigation into Cancer and Nutrition), que se inició en 1992 y se está realizando en 10 países europeos (Alemania, Dinamarca, España, Francia, Grecia, Países Bajos, Italia, Noruega, Reino Unido y Suecia) en 500000 mujeres y hombres voluntarios de edades comprendidas entre los 35 y 70 años, demostró dos hechos importantes: que el $50 \%$ de la población tiene un déficit parcial de micronutrientes; y que, en el $25 \%$ de la población, ese déficit es inferior al $50 \%$ de la cantidad diaria recomendada (CDR). Actualmente, se plantea la toma de suplementos orales para la mejora cutánea solo en situaciones de deficiencia, como el estilo vida sedentario, una dieta inadecuada, los trastornos del sueño, el tabaquismo, la polución excesiva o la menopausia. Se considera que la mejor estrategia nutricional para alcanzar un equilibrio entre la oxidación y la de antioxidantes naturales es el consumo regular de frutas y verduras.

Existe débil evidencia científica que relacione directamente al estrés psicológico con el envejecimiento cutáneo. Sí se ha demostrado relación con el deterioro de la barrera epidérmica y con el aumento de su permeabilidad.

También existe una relación científicamente demostrada entre dormir menos de 6 horas y el envejecimiento intrínseco. Y, asimismo, existe relación entre el aumento de temperatura y envejecimiento facial prematuro ${ }^{25}$. 
No obstante, actualmente, los estudios desarrollados en torno al exposoma y su influencia sobre el envejecimiento facial tienen algunos puntos débiles: los factores del exposoma han sido estudiados por separado, pero hay poco conocimiento sobre las interacciones entre ellos y sus consecuencias en el envejecimiento cutáneo. Es posible que su combinación genere efectos diferentes. La interacción entre los factores del exposoma y la genética justifica la individualización y la diferencia de respuesta entre individuos ante factores externos similares.

\section{TRATAMIENTO FACIAL DE ATRIBUTOS EMOCIONALES}

Cuando evaluamos el rostro de un paciente, el dermatólogo estético considera, en primer lugar, la salud cutánea y la ausencia o presencia de enfermedad en esa zona. Posteriormente, observa los signos de envejecimiento existentes a nivel facial, no solo de la piel externa, sino, además, de los compartimentos grasos, músculos y estructuras de soporte y retención facial, tanto en reposo como en movimiento, en visión frontal y lateral. Por último, establece las áreas de embellecimiento o perfeccionamiento que se puede obtener en el paciente según su edad y sexo.

Actualmente, adquiere enorme importancia para la dermatología estética la expresión facial de emociones $^{29}$, con objeto de que los tratamientos que instauremos permitan expresarlas adecuadamente. Al experimentar una emoción, podemos comunicar y expresar aquello que estamos sintiendo mediante cambios faciales, modificando nuestros gestos. Gran parte de la musculatura facial de los seres humanos se inserta en la piel y su contracción permite la gesticulación y, así, expresar lo que sentimos. Los seres humanos tenemos la capacidad de reconocer la expresión facial de las emociones. Si se muestran fotografías a personas, independientemente de la cultura o continente en el que vivan, identificarán la emoción que muestra el rostro. Y esto que parece simple revela estados mentales y psicológicos complejos, así como el resultado último de una actividad neuronal, muscular, vascular y bioquímica muy evolucionada. Se ha demostrado, además, que no solo nuestros sentimientos influyen en nuestros gestos, sino que nuestros gestos influyen en nuestros sentimientos. Todos sabemos que la risa nos hace sentir bien, pero no solo la risa natural nos hace sentir mejor, sino también la voluntaria, la desarrollada de forma consciente, no espontánea. Se liberan y modulan neurotransmisores y otros mediadores específicos ${ }^{30}$ que mejoran nuestro estado de ánimo, como la dopamina o el cortisol ${ }^{1}$.

En resumen, la expresión de las emociones y su reconocimiento en los rostros de otros seres humanos tienen un rol muy importante en el desarrollo y regulación de las relaciones interpersonales, con la formación de apegos (en la infancia y en las relaciones de pareja), así como en la regulación, el incremento o la disminución de conductas agresivas ${ }^{31}$.

El motivo actual por el que llegan muchas personas a nuestras consultas es mejorar su aspecto cuando este cambia debido al paso de los años. Este cambio tiene que ver tanto con la salud de nuestra piel, arrugas, surcos y volúmenes como con el reflejo emocional de nuestro rostro, el denominado reconocimiento facial emocional. Con los años, nuestro rostro muestra cambios anatómicos permanentes, similares a lo que observaríamos en los estados de cansancio, tristeza, enfado o flacidez, produciéndose una discordancia entre cómo nos sentimos y cómo lo reflejamos al mundo. En nuestra mano está mostrarles a nuestros pacientes todas las opciones que tenemos en dermatología estética para abordar estas necesidades desde la salud y la naturalidad. De las que más se utilizan por el excelente resultado que generan son las nuevas técnicas infiltrativas con ácido hialurónico de última generación.

Identificamos tres tipos de pacientes que, en lo referente a la estética facial, asisten a nuestras consultas:

- El primero es el que está preocupado por su piel y en el que se mezclan lo médico y lo estético 
dermatológico: manchas faciales, sensibilidad cutánea, lesiones o tumoraciones concretas, acné persistente ${ }^{9}$, cicatrices o tendencia a ellas, inflamación y enrojecimiento, vasos dilatados, etc. Tras un diagnóstico específico que descarte malignidad o premalignidad, en donde la epiluminiscencia (técnica basada en luz polarizada y sistemas de aumento) es fundamental, tenemos la opción de utilizar múltiples tratamientos ${ }^{6-8}$ : medicación oral, medicación tópica con o sin formulación magistral ${ }^{3}$, láser cutáneo (ablativo, no ablativo, vasculares o pigmentarios), luz pulsada médica, fluorescencia, leds, peelings químicos, plasma rico en plaquetas, mesoterapia...

- El segundo tipo de paciente busca embellecimiento, perfeccionar algunas zonas concretas. Suele ser joven y desea perfeccionar alguna zona facial, normalmente, relacionada con nuestras dos principales áreas de expresión: la zona periocular y la zona perilabial. El perfeccionamiento de los labios (perfilándolos, proyectándolos, voluminizándolos o hidratándolos), de la zona de las ojeras y de la nariz son las demandas más frecuentes. En hombres, la perfiloplastia, y la masculinización del mentón y del ángulo mandibular son solicitudes recurrentes. Y, sobre todo, tanto en mujeres como en hombres, la infiltración de toxina botulínica para relajar la musculatura de contracción: patas de gallo, y arrugas del entrecejo y de la frente.

- El tercer tipo de paciente que asiste a la consulta del dermatólogo estético es el paciente de rejuvenecimiento. Normalmente, expresa su deseo de forma concreta, mostrando la necesidad de solucionar la profundidad del surco nasogeniano, de las líneas de marioneta o las arrugas del entrecejo. Pero, curiosamente, lo que quiere no es lo que desea. Y no desea rejuvenecer años. Desea recuperar la capacidad de poder expresar con su rostro sus sentimientos. Con la edad, perdemos, unos antes que otros, la capacidad de expresar adecuadamente nuestros sentimientos y se nos instaura de forma fija un rictus diferente al que teníamos de más jóvenes. Parecemos enfadados, cansados o tristes sin estarlo. Y esta si- tuación tiene tratamiento con las técnicas infiltrativas de dermatología estética. El uso de productos tensores, voluminizadores, estimuladores y relajantes nos lo permiten.

\section{NUTRICIÓN EN DERMATOLOGÍA ESTÉTICA FACIAL}

Toda evidencia sobre nutrición es siempre compleja de obtener, por la dificultad de aislar una exposición a un determinado elemento del resto de variables nutricionales. Pero podemos hacer dos consideraciones claras: el deseo de los pacientes de controlar su sobrepeso como demanda frecuente en el paciente estético, tanto por razones estéticas como por salud; $y$, por otro lado, que hay cada vez mayor evidencia de la correlación existente entre elementos nutricionales concretos y el envejecimiento cutáneo ${ }^{32}$.

Tanto los niveles cuantitativos como los hábitos cualitativos en cuanto a macronutrientes, micronutrientes, oligoelementos o la propia agua parecen tener relación con los procesos de reparación, antioxidación, melanogénesis, colagenogénesis o el control del ambiente inflamatorio, de una forma similar a como sucede en los procesos del fotoenvejecimiento.

Estos procesos ocurren a través del aumento del estrés oxidativo (fig. 6), la producción de mutaciones o su reparación deficiente, la acumulación de depósitos de determinados productos de glicosilación, y un ambiente proinflamatorio mediado por citocinas.

Desde el punto de vista nutricional, estos procesos se pueden medir tanto de forma cuantitativa (peso, índice de masa corporal, valores antropométricos, niveles sanguíneos) como cualitativa (hábitos alimentarios, procesamiento de alimentos, exceso de determinadas grasas o azúcares); o el probable efecto de algunos agentes antioxidantes, cuyo uso en nutrición aplicada a la estética aún tiene evidencia escasa, difícil de evaluar y, por lo tanto, todavía deben desempeñar un rol complementario para el clínico ${ }^{33}$. 


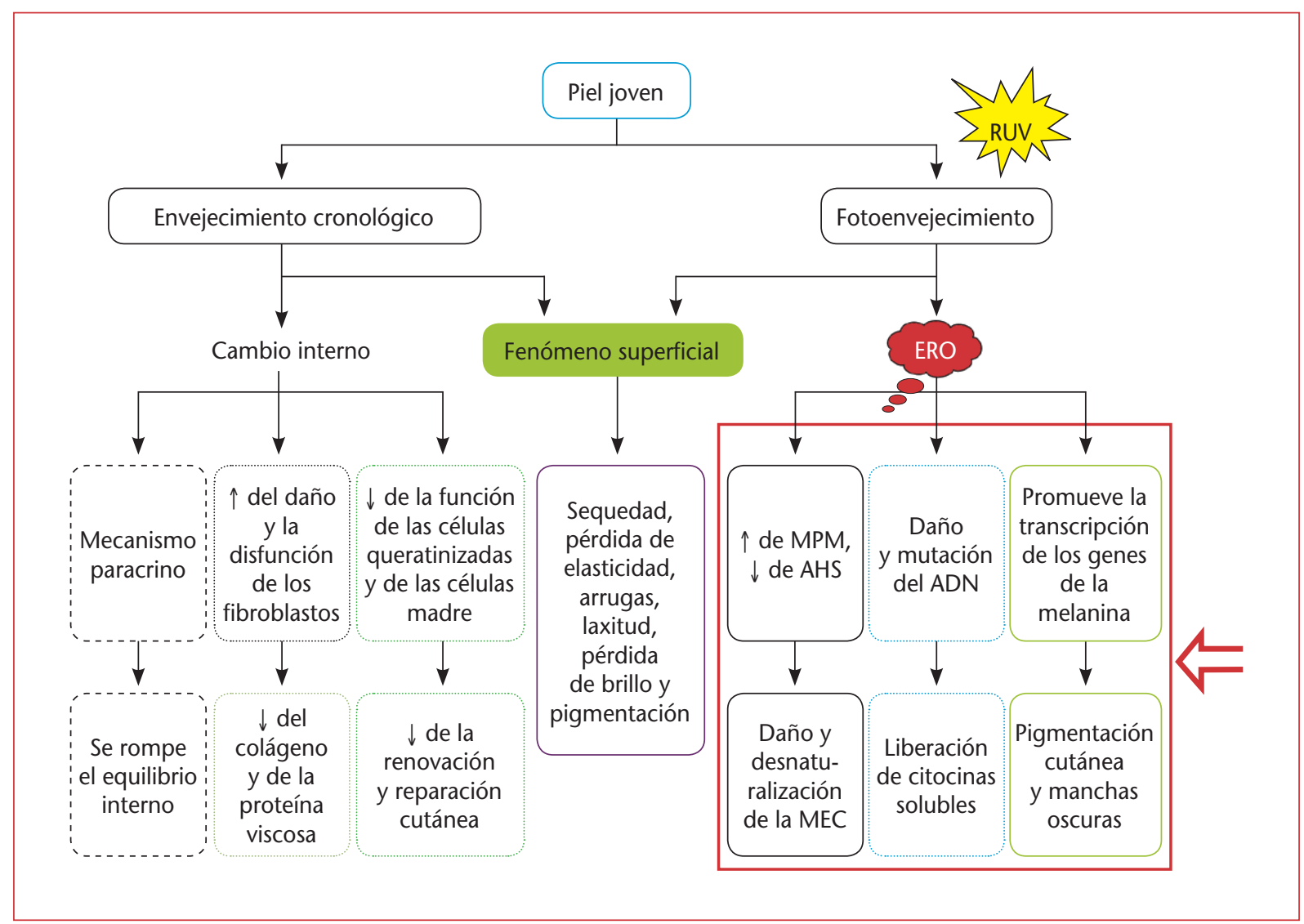

Figura 6. Envejecimiento extrínseco (derecha) y mecanismos implicados.

ADN: ácido desoxirribonucleico; AHS: ácido hialurónico-sintasa; ERO: especies reactivas del oxígeno; MEC: matriz extracelular; MPM: metaloproteinasas de la matriz; RUV: radiación ultravioleta.

Traducida de: Cao C, Xiao Z, Wu Y, Ge C. Diet and skin aging-from the perspective of food nutrition. Nutrients. 2020;12(3):870.

Para tratar de compensar el envejecimiento influido por la dieta, nuestra intervención nutricional tiene por objetivo la mejora cuantitativa y cualitativa en la alimentación y los hábitos del paciente ${ }^{34}$ mediante:

- Modificación de sus hábitos alimentarios cuantitativos y cualitativos.

- Programa de ejercicio físico adecuado a las posibilidades del paciente.

Estos dos primeros aspectos siguen siendo los pilares fundamentales sin los cuales ninguna de las otras estrategias resultará efectiva:

- Estrategias de manejo del estrés y la motivación.
- Y, especialmente en los últimos años, valoraremos usar apoyo farmacológico, cuando el eje de intervención sobre el paciente sea el control del sobrepeso o la obesidad.

Dentro del uso de fármacos para el control del exceso de peso, hasta hace poco tiempo, solo se disponía del orlistat como fármaco aprobado para esta indicación y con evidencia de eficacia, si bien, únicamente actúa a nivel intestinal, reduciendo la absorción de grasas de cada ingesta y, con ello, disminuyendo la ingesta calórica, sin actuar sobre otros aspectos de la nutrición, como el metabolismo de los hidratos de carbono o la sensación de saciedad, entre otros. 
En los últimos años, han aparecido dos herramientas farmacológicas más:

- Combinaciones de antidepresivos con antagonistas de opiáceos como la combinación de buprenorfina y naloxona, que trata básicamente de regular la sensación de saciedad y la ingesta compulsiva. No obstante, esta vía no está exenta de efectos sobre las funciones cerebrales superiores, que pueden provocar frecuentes efectos adversos en esa esfera y, por lo tanto, ser excesivamente intrusivos como tratamiento para este fin.

- Análogos del péptido similar al glucagón de tipo 1 (GLP-1; del inglés, glucagon-like peptide-1): liraglutida. Este fármaco usa una nueva vía de intervención a través de la regulación de la sensación de saciedad tanto a nivel intestinal ${ }^{35}$ como en el eje hipotálamo-hipofisario, en lo cual nos vamos a detener con más detalle.

El GLP-1 y el péptido inhibidor gástrico (GIP; del inglés, gastric inhibitory peptide) modulan fisiológicamente en tiempo real la secreción de insulina, en parte, por su semivida de pocos minutos. Entre otros efectos, inducen sensación de saciedad a través del eje hipotálamo-hipofisario y del retraso del vaciamiento gástrico. La liraglutida tiene estos mismos efectos, pero con una semivida de horas, lo que parece convertirla en una herramienta terapéutica que considerar para el sobrepeso y la obesidad, trastornos mediados por los mismos hábitos alimentarios que incrementan el estrés oxidativo y empeoran el aspecto estético de la piel.

Mejorando este ambiente oxidativo, es esperable una reducción del daño vascular ${ }^{36}$, especialmente, en la microcirculación cutánea y, secundariamente, una mejora en el aspecto estético de la piel $^{37}$, ya que una piel sana es esencialmente una piel estética.

\section{BIBLIOGRAFÍA}

1. Santoro-Lamelas V. La salud pública en el continuo saludenfermedad: un análisis desde la mirada profesional. Rev Salud Pública. 2016;18(4):230-7.
2. Almeida-Filho N. Para una teoría unificada sobre salud-enfermedad: I. Salud como objeto-modelo complejo. Rev Saúde Públ. 2013;47(3):433-50.

3. Bagatin E, Florez-White M, Arias-Gomez MI, Kaminsky A. Algorithm for acne treatment: Ibero-Latin American consensus. An Bras Dermatol. 2017;92(5):689-93.

4. Kaminsky A, Florez-White M, Bagatin E, Arias Ml; Iberian Latin American Acne Studies Group (GILEA - Grupo Ibero-Latinoamericano de Estudio del Acne). Large prospective study on adult acne in Latin America and the lberian Peninsula: risk factors, demographics, and clinical characteristics. Int J Dermatol. 2019;58(11):1277-82.

5. Rodríguez de Romo AC. Claude Bernard, el hombre y el científico. An Med (Mex). 2007;52(2):90-6.

6. Peñuela-Olaya MA. Una visión humanista sobre el campo de la salud. Perinat Reprod Hum. 2010;24(4):265-71.

7. García-Gavín J, Eguren-Michelena C; Grupo de Salud Integral de la Piel. Dermatología ¿estética? Actas Dermosifilogr. 2021;112:679-81.

8. Perales-Cabrera A. Ética y humanismo en la formación médica. Acta Bioeth. 2008; 14(1):30-8.

9. Torres H, Torres X. Situación de la cosmetología y de la estética dentro de la dermatología. Evolución dermocosmética en los últimos 30 años. Med Cután Ibero-Latino-Am. 2005; 33(1):1-5.

10. Tamas T, Dinu C, Lenghel M, Băciut $G$, Bran $S$, Stoia $S$, et al. The role of ultrasonography in head and neck non-melanoma skin cancer approach: an update with a review of the literature. Med Ultrason. 2021;23(1):83-8.

11. Cral WG. Ultrasonography and facial aesthetics. Aesthetic Plast Surg. 2021. [En prensa].

12. Mlosek RK, Migda B, Migda M. High-frequency ultrasound in the 21st century. J Ultrason. 2021;20(83):e233-41.

13. Alfageme F, Wortsman $X$, Catalano O, Roustan G, Crisan M, Crisan D, et al. European Federation of Societies for Ultrasound in Medicine and Biology (EFSUMB) Position Statement on Dermatologic Ultrasound. Ultraschall Med. 2021;42(1): 39-47.

14. Dopytalska K, Sobolewski P, Mikucka-Wituszyńska A, Gnatowski M, Szymańska E, Walecka I. Noninvasive skin imaging in esthetic medicine-Why do we need useful tools for evaluation of the esthetic procedures. J Cosmet Dermatol. 2021; 20(3):746-54.

15. Massufero Vergilio M, Idalgo Vasques L, Ricci Leonardi G. Characterization of skin aging through high-frequency ultrasound imaging as a technique for evaluating the effectiveness of anti-aging products and procedures: a review. Skin Res Technol. 2021. [En prensa].

16. Alfageme F, Cerezo E, Aguiló R. Manual de ecografía cutánea. CreateSpace Independent Publishing Platform; 2013.

17. Malinowska S, Jaguś D, Woźniak W, Mlosek RK. Usefulness of high-frequency ultrasound in the monitoring of laser treatment of acne scars. J Ultrason. 2021;20(83):e279-83.

18. Jaguś D, Skrzypek E, Migda B, Woźniak W, Mlosek RK. Usefulness of Doppler sonography in aesthetic medicine. J Ultrason. 2021;20(83):e268-72.

19. Li Z, Yang Y, Yu N, Zhou W, Li Z, Chong Y, et al. The "visible" muscles on ultrasound imaging make botulinum toxin injection more precise: a systematic review. Aesthetic Plast Surg. 2021. [En prensa]. 
20. Mashkevich G, Wang J, Rawnsley J, Keller GS. The utility of ultrasound in the evaluation of submental fullness in aging necks. Arch Facial Plast Surg. 2009;11(4):240-5.

21. De Cabo-Francés FM, Alcolea JM, Bové-Farré I, Pedret C, Trelles MA. Ultrasound of injectable fillers and its role in diagnosis tracking. Cir Plást Iberolatinoam. 2012;38(2):179-87.

22. Urdiales-Gálvez F, De Cabo-Francés FM, Bové I. Ultrasound patterns of different dermal filler materials used in aesthetics J Cosmet Dermatol. 2021;20(5):1541-8.

23. Stefanovic N, Irvine AD, Flohr C. The role of the environment and exposome in atopic dermatitis. Curr Treat Options Allergy. 2021;1-20. [En prensa].

24. Vermeulen R, Schymanski EL, Barabási AL, Miller GW. The exposome and health: where chemistry meets biology. Science. 2020;367(6476):392-6.

25. Passeron T, Zouboulis CC, Tan J, Andersen ML, Katta R, Lyu $X$, et al. Adult skin acute stress responses to short-term environmental and internal aggression from exposome factors. J Eur Acad Dermatol Venereol. 2021;35(10):1963-75.

26. Passeron T, Krutmann J, Andersen ML, Katta R, Zouboulis CC. Clinical and biological impact of the exposome on the skin. J Eur Acad Dermatol Venereol. 2020;34 Suppl 4:4-25.

27. Khmaladze I, Leonardi M, Fabre S, Messaraa C, Mavon A. The skin interactome: a holistic "genome-microbiome-exposome" approach to understand and modulate skin health and aging. Clin Cosmet Investig Dermatol. 2020;13:1021-40.

28. Krutmann J, Schalka S, Watson REB, Wei L, Morita A. Daily photoprotection to prevent photoaging. Photodermatol Photoimmunol Photomed. 2021. [En prensa].
29. De Maio M. MD Codes ${ }^{\mathrm{TM}}$ : a methodological approach to facial aesthetic treatment with injectable hyaluronic acid fillers. Aesth Plast Surg. 2021;45(2):690-709.

30. Yim JE. Therapeutic benefits of laughter in mental health: a theoretical review. Tohoku J Exp Med. 2016;239(3):243-9.

31. Cordaro DT, Sun R, Kamble S, Hodder N, Monroy M, Cowen A, et al. The recognition of 18 facial-bodily expressions across nine cultures. Emotion. 2020;20(7):1292-300.

32. Cao $C, X i a o, Z, W u Y, G e C$. Diet and skin aging-from the perspective of food nutrition. Nutrients. 2020;12(3):870.

33. Alfageme Roldán F. Ecografía del envejecimiento cutáneo y de la reestructuración dérmica. Más Dermatol. 2021;34: 29-33.

34. Grannell A, Martin WP, Dehestani B, Al-Najim W, Murphy JC, le Roux CW. Liraglutide does not adversely impact fat-free mass loss. Obesity (Silver Spring). 2021;29(3):529-34.

35. Charpentier J, Briand F, Lelouvier B, Servant F, Azalbert V, Puel $A$, et al. Liraglutide targets the gut microbiota and the intestinal immune system to regulate insulin secretion. Acta Diabetol. 2021;58(7):881-97.

36. Nowrouzi-Sohrabi P, Soroush N, Tabrizi R, Shabani-Borujeni $M$, Rezaei S, Jafari F, et al. Effect of liraglutide on cardiometabolic risk profile in people with coronary artery disease with or without type 2 diabetes: a systematic review and metaanalysis of randomized controlled trials. Front Pharmacol. 2021;12:618208.

37. Thijssen DHJ, Carter SE, Green DJ. Arterial structure and function in vascular ageing: are you as old as your arteries? J Physiol. 2016;594(8):2275-84. 